\title{
Convection in brown dwarfs
}

\author{
Subhanjoy Mohanty ${ }^{1}$, Isabelle Baraffe ${ }^{2}$ \\ and Gilles Chabrier ${ }^{2}$ \\ ${ }^{1}$ Harvard-Smithsonian Center for Astrophysics, Cambridge, MA 02138, USA \\ email: smohanty@cfa.harvard.edu \\ ${ }^{2}$ CRAL, École Normale Supérieure, 46 allée d'Italie, 69007 Lyon, FRANCE \\ email: [ibaraffe; chabrier]@ens-lyon.fr
}

\begin{abstract}
We briefly review the crucial effect that convection plays in the evolution, interior and atmospheric physics of brown dwarfs. In particular, we discuss: (1) the role of convection in the interior structure and evolution of brown dwarfs (BDs); (2) the importance of convection in the atmospheres of ultra-cool objects, at both high and low gravities (i.e., in both young and old BDs); and (3) the role of convection/turbulence in the formation and settling of dust grains, and non-equilibrium chemistry, in BD atmospheres.
\end{abstract}

Keywords. Stars: brown dwarfs, convection

\section{Introduction}

Brown dwarfs are by definition substellar objects: with a mass less than about 75 Jupiters, they are simply to small to sustain stable hydrogen fusion. Consequently, they have no Hydrogen-burning Main Sequence phase. After an initial period of Deuterium fusion $\dagger$, they simply cool down and grow fainter with time. Eventually, their cores become degenerate, supported by electron degeneracy pressure. Astronomers have discovered hundreds of these diminutive bodies in the last few years, both in the field as well as in star-forming regions and young clusters; indeed, it now appears that their number densities are comparable to those of low-mass stars (Chabrier 2003), making brown dwarfs one of the major components of our galaxy (in number, though not in mass).

The study of these substellar objects has become one of the hot topics in stellar and planetary astrophysics, for a number of reasons. Their formation mechanism remains somewhat of a mystery, with important attendant implications for the general theory of star formation and the origins of the low-mass stellar IMF (Reipurth \& Clarke 2001; Bate et al. 2003; Padoan \& Nordlund 2004). They also offer keen insights into the physics of compact objects. Their ultra-cool atmospheres moreover are similar to those of giant planets, with various exotic phenomena such as the formation of complex molecules, appearance and eventual settling of dust grains, formation of clouds, and precipitation. While a number of extra-solar giant planets (EGPs) have been discovered so far, their direct observation remains mostly out of the reach of current technology (due to the overwhelming light of the central host star); consequently, free-floating brown dwarfs presently offer some of the best insights into planetary atmospheres and evolution. Finally, in the context of this article, they offer us the unique opportunity to study the behavior of convection in both optically thick and thin regimes. This is not only important for a general understanding of convective processes, but also has crucial implications for the atmospheric and evolutionary properties of BDs, and by extension, those of EGPs.

$\dagger$ In brown dwarfs with mass greater than about 12 Jupiters; in less massive ones, the so-called 'planetary-mass' brown dwarfs, there is no D-fusion either. 
In this article, we present a general overview of convection in brown dwarfs from three different standpoints: (1) general convective properties of older (field, higher-gravity) brown dwarfs; (2) effect of convection on the evolution of young (low-gravity) brown dwarfs; and (3) role of convection in brown dwarf atmospheres (overshoot, dust settling, dredging). We stress that the discussion here will be confined to convection within the context of Mixing Length Theory (MLT); detailed 3-D radiative hydrodynamic (RHD) simulations are examined in the article by H.-G. Ludwig in this volume.

\section{Convection in field brown dwarfs}

The role of superadibaticity is well known in the atmospheres of solar-type stars. In objects with mass $\lesssim 0.6 \mathrm{M}_{\odot}\left(\mathrm{T}_{\text {eff }} \lesssim 5000 \mathrm{~K}\right)$, however, the situation changes. In these objects, $\mathrm{H}_{2}$ forms efficiently, leading to two effects: a much larger atmospheric opacity due to $\mathrm{H}_{2}$ collisionally induced absorption (CIA), and thus a suppresion of radiative flux $\mathrm{F}_{\text {rad }}$; and a decrease in the adiabatic gradient, due to an increase in the specific heat capacity $\left(c_{p}\right)$ arising from the larger number of internal degrees of freedom in molecules. Both effects favor efficient convection (Chabrier \& Baraffe 2000). As a result, the extent of superadiabatic layers are much diminished at these masses, and convection is nearly perfectly adiabatic, even into optically thin layers. The fully convective to fully radiative jump occurs sharply, with only a small entropy jump. These predictions, made employing MLT, are confirmed by 3-D simulations as well (e.g., Ludwig 2006).

On the one hand, efficient convection removes some of the uncertainties of MLT, since the choice of mixing-length is almost immaterial. On the other hand, strong convection at $\tau<1$ means that the transfer equations for the radiative and conductive fluxes $\left(\mathrm{F}_{\mathrm{rad}}\right.$ and $\left.\mathrm{F}_{\text {conv }}\right)$ must be solved simultaneously in the atmosphere, instead of $\mathrm{F}_{\text {rad }}$ equations alone. The strongly wavelength-dependent photospheric opacities at these $T_{\text {eff }}$ further implies that the transfer equations must be solved with self-consistent non-grey atmospheres, the neglect of which leads to incorrect mass-temperature and mass-luminosity relationships. For masses below $\sim 0.6 \mathrm{M}_{\odot}$, i.e., for very low mass stars (VLMS), BDs and EGPs, this has been the prime focus of evolutionary and atmospheric modeling.

In this context, we note that objects with mass $\lesssim 0.3 \mathrm{M}_{\odot}$ (spectral types $\gtrsim \mathrm{M} 3$ in the field), i.e., the lowest mass stars as well as all BDs, are fully convective, without any radiative core.

Finally, we provide an interesting sidelight in this regard on giant planets; in particular, the effect of alkali metals on convection in their interiors. For pressures $\gtrsim 1$ bar and temperatures $\lesssim 1000 \mathrm{~K}$, the 3 main opacity sources are $\mathrm{H}_{2} \mathrm{O}, \mathrm{CH}_{4}$, and $\mathrm{H}_{2}$-CIA. The opacity rises with increasing pressure at these temperatures, ensuring fully convective conditions. However, for temperatures $\sim 1200-1500 \mathrm{~K}, \mathrm{H}_{2}$ and $\mathrm{He}$ opacities behave differently: their absorption at any given wavelength increases with density, but since the temperature also rises, the photons are emitted at shorter wavelengths, where the monochromatic absorption is smaller. Consequently, the opacity can decrease, potentially causing a deep radiative zone in giant planet interiors. However, BD atmospheres show that there is very strong absorption by $\mathrm{Na}$ I and $\mathrm{K}$ I alkali line-wings in regions where $\mathrm{H}_{2} \mathrm{O}, \mathrm{CH}_{4}, \mathrm{NH}_{3}, \mathrm{H}_{2}$ and $\mathrm{He}$ are almost transparent. The increased opacity due to the alkalis (if they are present in giant planet interiors) removes the radiative zone and allows convection everywhere (Guillot et al. 2006 and references therein). Fig. 1 illustrates these effects for Jupiter. 


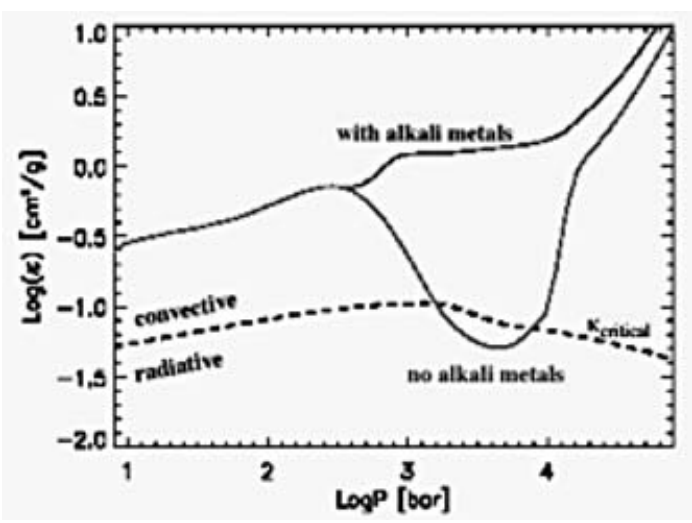

Figure 1. Comparison of Roseland mean opacities (solid lines) along a model of Jupiter to the critical opacity (dashed line) above which the medium is convectively unstable. The opacity calculations assume a three times solar enrichment in heavy elements. Depending on the presence of alkali metals, Jupiter is found to be fully convective (top solid curve) or to possess a small deep radiative zone (dip in bottom solid curve). [From Guillot et al. 2006].

\section{Effect of convection on initial brown dwarf evolution}

We have seen above that field objects with mass $\lesssim 0.6 \mathrm{M}_{\odot}$ have efficient convection, with negligible dependence on the mixing-length. The situation is very different, however, for very young objects, even with mass $\lesssim 0.6 \mathrm{M}_{\odot}$, because their gravities are much lower. This is because of the reverse of the process discussed above: $\mathrm{H}_{2}$ formation decreases with lower gravity (i.e., lower pressure) for a given temperature.

As mentioned above, $\mathrm{H}_{2}$ formation yields more efficient convection, both by increasing opacity due to $\mathrm{H}_{2}$-CIA, and producing a lower adiabatic gradient. The latter produces a flatter T-P profile, i.e., lower temperature for a given pressure in the hotter regions. This in turn provokes even more $\mathrm{H}_{2}$ formation, and thus still more efficient convection, and so on. In other words, the atmospheric T-P profile has a strongly non-linear response to the onset of $\mathrm{H}_{2}$ formation. Since the onset of $\mathrm{H}_{2}$ in turn depends on the gravity (Fig. 2a), the atmospheric T-P profile is a strong function of gravity too (Baraffe et al. 2002).

As a result, the initial conditions one adopts can severely affect the subsequent evolution of BDs. In particular, the initial gravity, i.e., the starting radius for a BD of given mass, one assumes is crucial for the evolution. Fig. $2 \mathrm{~b}$ shows the difference in evolution for BDs assuming a starting gravity of $\log g=2.5$ versus $\sim 3.5$ (c.g.s. units). In the case of the lower initial gravity, $\mathrm{T}_{\text {eff }}$ initially increases with time for a given mass BD: this is because gravity increases with time, hence more $\mathrm{H}_{2}$ forms, leading to increasingly efficient convection, and thus hotter $\mathrm{T}_{\text {eff }}$ (since interior $\mathrm{T}-\mathrm{P}$ profile is fixed). Note that BDs with initial $\mathrm{T}_{\text {eff }} \approx 2200-4000 \mathrm{~K}$ are affected most severely by the different initial gravities. For $\mathrm{T}_{\text {eff }} \gtrsim 4000 \mathrm{~K}, \mathrm{H}_{2}$ does not form in significant quantities, while for $\mathrm{T}_{\text {eff }}$ $\lesssim 2200 \mathrm{~K}$, the outer layers are dense and cool enough for efficient $\mathrm{H}_{2}$ formation even at $\log g=2.5$; in either case, the cascade of effects affected with the onset of $\mathrm{H}_{2}$ formation does not arise, unlike in the intermediate temperature BDs.

Similarly, BD evolution is also strongly affected by the mixing-length adopted, since this too affects the formation efficiency of $\mathrm{H}_{2}$ (larger mixing-length producing more $\mathrm{H}_{2}$; see Fig. 2a), with all the attendant effects discussed above.

Finally, we point out that differences in the evolutionary path caused by different initial conditions are largely gone by ages of $\sim 1$-few Myrs. That is, the initial conditions are 'forgotten' after this period (Fig. $2 \mathrm{~b}$ illustrates this for the different initial gravities). 

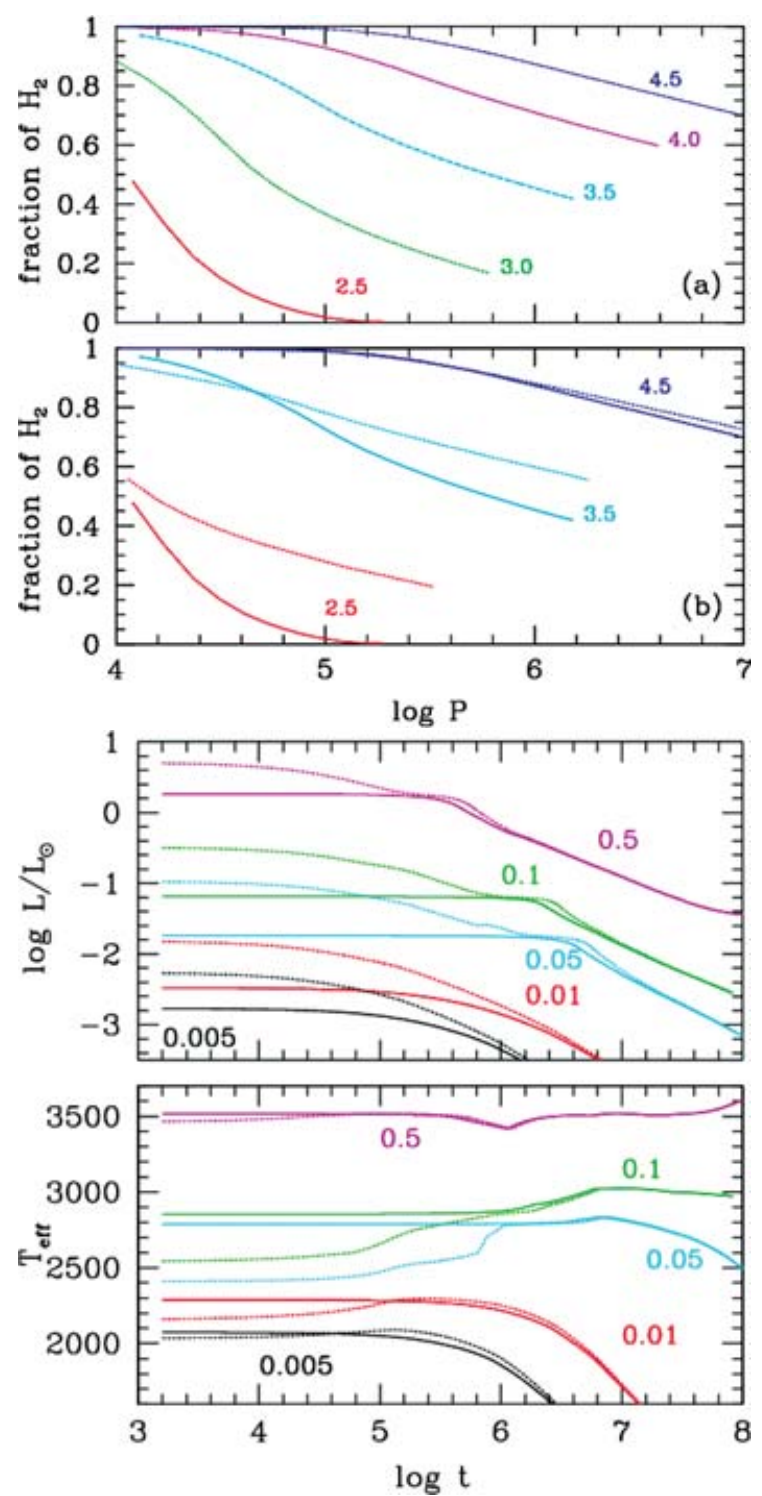

Figure 2. Top two panels (Fig. 2a): Number fraction of molecular $\mathrm{H}_{2}$ as a function of pressure (dyne $/ \mathrm{cm}^{2}$ ), for various gravities (labeled in $\log g$, cgs), at $T_{\text {eff }}=2500 \mathrm{~K}$. The top panel shows the curves assuming mixing length $=1 \mathrm{H}_{P}$; the panel below assumes either mixing-length $=1$ $\mathrm{H}_{P}$ (lower curve in every log $g$ pair) or $2 \mathrm{H}_{P}$ (higher curve in every $\log g$ pair). Bottom two panels (Fig. 2b): Effect of initial radius on the evolution of luminosity (top panel) and $\mathrm{T}_{\text {eff }}$ (bottom panel) as a function of time (log yrs) for several masses (indicated in plot, in $\mathrm{M}_{\odot}$ ). Solid lines show curves for initial $\log g \sim 3.5$, and dashed lines show initial $\log g=2.5$. The mixing-length assumed is $1 \mathrm{H}_{P}$. [From Baraffe et al. 2002].

Thus, current evolutionary models - which have no good empirical knowledge of initial conditions and thus choose them rather arbitrarily - are nevertheless good for comparisons to BD data for ages $\gtrsim 1-$ few Myrs. For ages $\lesssim 1$ Myr, however, conclusions about $\mathrm{BD}$ properties from comparisons to evolutionary models are meaningless at present. Better constraints on initial conditions (e.g., initial size, accretion effects, appropriate mixing length) are sorely needed to characterize the youngest BDs. The interested reader may 
examine Baraffe et al. (2002), and references therein, for a fuller description of these evolutionary issues and uncertainties at young ages.

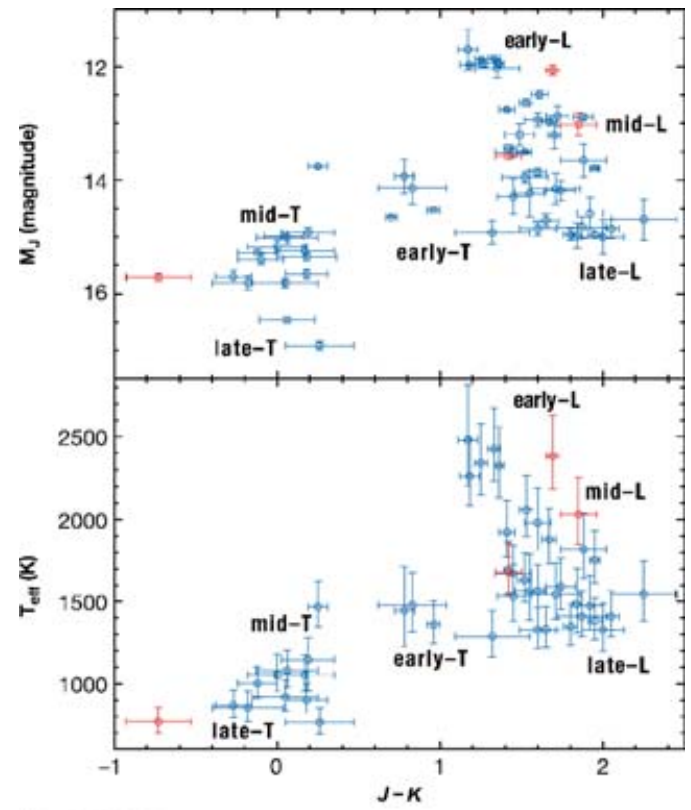

Kinkpariak JD 3005

Ana Rev. Astroa. Astrogleys. 43. 195-24s

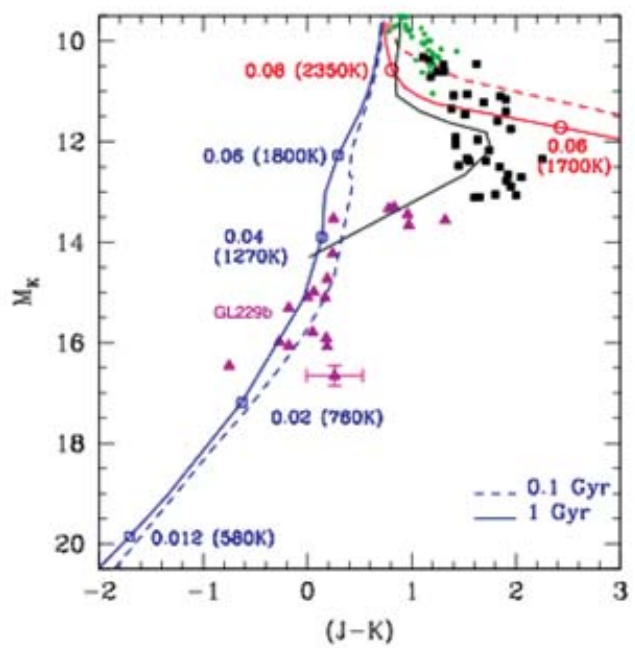

Figure 3. Top two panels (Fig. 3a): Absolute J-mag (top panel), or equivalently, $\mathrm{T}_{\text {eff }}$ (bottom panel), as a function of $J-K$ color for L and T dwarfs. From early $\mathrm{L}$ to late T, a clear drift is visible first to redder colors and then a sharp turn bluewards. [From Kirkpatrick 2005]. Bottom panel (Fig. 3b): Absolute $K$-mag vs. $J-K$ color for M, L and T dwarfs, compared to synthetic spectra predictions. The two rightmost curves are predictions assuming no-settling (DUSTY models); the two leftmost curves are assuming full-settling (COND models). The middle curve is assuming gradual settling (SETTL models), and matches the data, especially in the settling L-T transition regime, much better. [From Chabrier 2005]. 


\section{Role of convection in brown dwarf atmospheres}

Going from $\mathrm{M}$ type objects to L-types, and thence to T-types (i.e., decreasing $\mathrm{T}_{\text {eff }}$ ), one finds that the near-infrared (NIR) $J H K$ spectrum first turns red, but then turns blue again. This is easily seen in the $J-K$ colors as well (Fig. 3a). The redward trend is predominantly due to the formation of dust grains in the atmosphere, while the blueing is due to the settling of these grains below the photosphere, as well as the onset of strong $\mathrm{CH}_{4}$ opacity in the $K$-band (see Kirkpatrick 2005, and references therein). Now, even though the convective layer has sunk beneath the photosphere by the L-types, the top of the convection zone is still within $\sim 1$-few pressure scale-heights of the photosphere (e.g., Allard et al. 2001). As a result, the settling of grains is strongly dependent on the competition between the relevant timescales for mixing by convective overshoot, gravitational settling, dust formation and dust destruction. MLT by definition cannot handle overshoot, so full 3-D RHD simulations are required to treat this problem rigorously. Such simulations are discussed in the contribution by H.-G. Ludwig, so we do not describe them here; however, we show some intial results based on such simulations. Fig. $3 \mathrm{~b}$ shows that synthetic spectra incorporating gradual settling based on general implications of the RHD simulations are in much better agreement with the observed colors of field VLMS and BDs - and in particular, are beginning to better confront the observations in the L-T transition regime where settling is ongoing - than spectra based on unrealistic no-settling or full-settling assumptions (e.g., Chabrier et al. 2005).
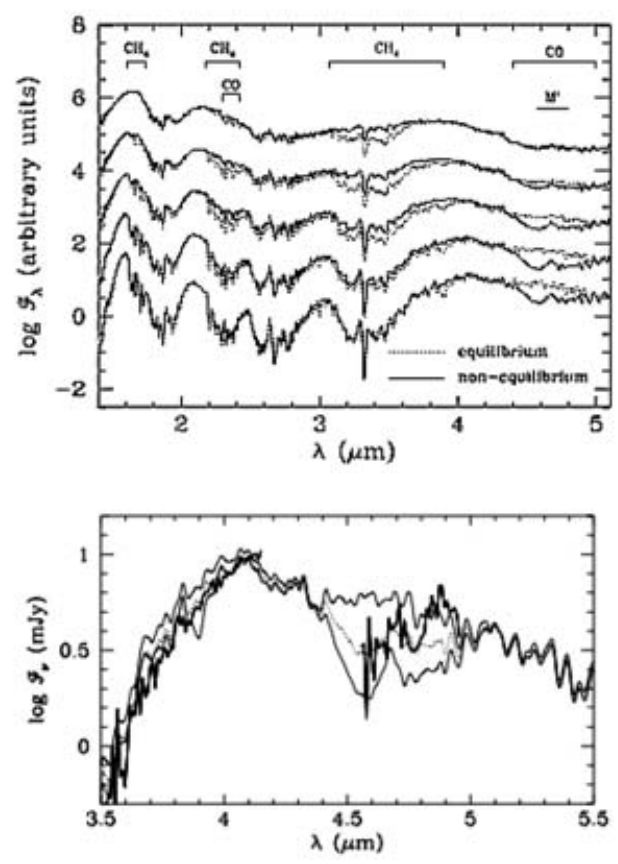

Figure 4. Top panel (Fig. 4a): A comparison of spectra computed with abundances from chemical equlibrium (dashed lines) and with non-equilibrium abundances resulting from vertical transport (solid lines). All models have $\log g=5.0$; $\mathrm{T}_{\text {eff }}$ decreases from $1600 \mathrm{~K}$ to $800 \mathrm{~K}$ (top to bottom) in steps of $200 \mathrm{~K}$. Main bands of $\mathrm{CO}$ and $\mathrm{CH}_{4}$ and the bandpass of the $M^{\prime}$ filter are indicated. Bottom panel (Fig. 4b): CO in Gl 229b. Observed spectrum shown as thick solid line, compared to models with $\mathrm{T}_{\text {eff }}=1000 \mathrm{~K}, \log g=5.0$. The models depart increasingly from chemical equilibrium, with the top curve being at equilibrium and the bottom one the furthest from it. [From Saumon et al. 2003]. 
Finally, convective overshoot mixing can also lead to non-equilibrium chemistry in BD atmospheres. For example, much higher CO opacity is seen in the 4-5 $\mu \mathrm{m}$ region of the spectrum in the BD Gl 229b than expected from chemical equilibrium calculations for its $\mathrm{T}_{\text {eff }}$ (which favor the formation of $\mathrm{CH}_{4}$ ). This is thought to be due to dredging up of $\mathrm{CO}$ from the hotter layers below, by convective overshoot motions, on a shorter timescale than its conversion to $\mathrm{CH}_{4}$. Fig. 4a shows predicted changes in opacity, due to such convection-induced non-equilibrium chemistry; Fig. 4b shows that such predictions are indeed more consistent with Gl 229b's spectrum than equilibrium calculations neglecting the effects of convection (Saumon et al. 2003).

\section{Conclusions}

The discussion above indicates that convection has extremely important effects on the intrinsic properties of BDs, their evolution and their atmospheres, at both young and old ages. While MLT theory has been employed so far to treat BD convection, full 3-D RHD simulations are sorely needed to provide guidance in the unique conditions on BDs (and EGPs): in particular, to treat convection in optically thin layers and the behaviour of overshoot motions. Such simulations are now coming to the fore, and should significantly improve our modeling, and thus understanding, of BDs in coming years. Finally, we note that a number of recent studies have drawn conclusions about the masses and other properties of extremely young (age $<1 \mathrm{Myr}$ ) BDs, based on current theoretical evolutionary tracks. While the results are potentially exciting (e.g., the existence of 'planetary-mass' BDs), they must be treated with extreme caution, due to the very large uncertainties in these tracks at early ages arising from our ignorance of initial conditions.

\section{Acknowledgements}

S.M. is grateful to the Spitzer Fellowship for funding this research; to the IAU S239 SOC for inviting him for the talk; and to H.-G. Ludwig, F. Allard, and G. Basri for very helpful discussions on $\mathrm{BD}$ convection and atmospheres.

\section{References}

Allard, F., Hauschildt, P., Alexander, D., Tamanai, A. \& Schweitzer, A. 2001, ApJ 556,357

Baraffe, I., Chabrier, G., Allard, F. \& Hauschildt, P. 2002, A\& A 382, 563

Bate, M., Bonnell, I. \& Bromm, V. 2003, MNRAS 339, 577

Chabrier, G. \& Baraffe, I. 2000, PASP 115, 763

Chabrier, G. 2003, PASP 115, 763

Chabrier, G., Baraffe, I., Allard, F. \& Hauschildt, P. 2005, ASP Conference Series astro$\mathrm{ph} / 0509798$

Guillot, T., Stevenson, D., Hubbard, W. \& Saumon, D. 2006, PASP 115, 763

Kirkpatrick, D. 2005, ARAA 43, 195

Ludwig, H.-G. 2006, $A \& A$ accepted, astro-ph/0608264

Padoan, P. \& Nordlund, Å. 2004, ApJ 617, 559

Reipurth, B. \& Clarke, C. 2001, AJ 122, 432

Saumon, D., et al. 2005, ASP Conference Series astro-ph/0207070

\section{Discussion}

Montalbán: Do you follow deuterium burning in your models? Because I think that if your models are different depending on the initial $\log g$, this is because your models with $\log g=3$ are not able to follow the D-burning in equilibrium. 
Mohanty: The models do follow D-burning: In both sets of models, D-burning is accounted for once it starts. This is why the $T_{\text {eff }}$ and luminosity level out over a period of time (during ongoing D-burning), before decreasing again once the $\mathrm{D}$ is exhausted. The point is simply that there is no good definition of what the starting point should be, i.e., at what $\log g$ should $t=0$ be defined, for comparison to observations.

CHIosi: Can you expand the argument of rapid settling of grains? I imagine that some timescale is redefined?

Mohanty: I did not mean to imply that in the COND models, the grains are modeled to settle out extremely fast. What I meant is that the grains are simply assumed to have 'already settled out' in the COND models; thus, these models do not take any dust opacity into account (though they do account for the depletion of grain-forming species).

SimON: Can one use these results to distinguish among formation mechanisms for brown dwarfs of different mass? For example, to distinguish at which mass pure collapse is most important and when accretion becomes important?

MOHANTY: I think this will be possible at some point; however, detailed modeling of the effects of accretion on the initial evolution (now underway by Chabrier, Baraffe and collaborators, among others) will be required before we can do this. 